\title{
Clinicopathological and prognostic significance of circulating tumor cells in patients with esophageal cancer: a meta-analysis
}

This article was published in the following Dove Press journal:

OncoTargets and Therapy

\author{
Jinxuan $\mathrm{Hou}^{1,2, *}$ \\ Kun Zou ${ }^{2, *}$ \\ Chaogang Yang ${ }^{2}$ \\ Xiaohua Leng' \\ Yu Xu $u^{1,3}$
}

'Hubei Key Laboratory of

Tumor Biological Behaviors and

Hubei Cancer Clinical Study

Center, Zhongnan Hospital of Wuhan University, Wuhan, China;

${ }^{2}$ Department of Thyroid and Breast Surgery, Zhongnan Hospital of

Wuhan University, Wuhan, China;

${ }^{3}$ Department of Radiation and Medical

Oncology, Zhongnan Hospital of

Wuhan University, Wuhan, China

*These authors contributed equally to this work
Correspondence: Yu Xu

Department of Radiation and Medical Oncology, Zhongnan Hospital of Wuhan

University, Hubei Key Laboratory

of Tumor Biological Behaviors and

Hubei Cancer Clinical Study Center,

169 Donghu Road, Wuchang District,

Wuhan 43007I, China

Tel +86 $27678|3| 55$

Fax +86 2767812892

Email xuyu@whu.edu.cn
Background: The aim of this meta-analysis was to assess the clinicopathological and prognostic significance of circulating tumor cells (CTCs) in patients with esophageal cancer (EC).

Methods: We searched PubMed, EMBASE, Science Citation Index Expanded, Cochrane library (from inception to July 2018) with the keywords "esophageal cancer", "circulating tumor cells", "prognosis", and "peripheral blood". HR, risk ratio (RR), OR, and their 95\% CIs were set as effect measures. All analyses were performed by STATA 12.0.

Results: Eighteen studies were retrieved; CTC-positive patients were significantly associated with poor progression-free survival (PFS) $(\mathrm{HR}=2.61$; 95\% $\mathrm{CI}=2.08-3.28)$ and overall survival (OS) $(\mathrm{HR}=2.50 ; 95 \% \mathrm{CI}=2.12-2.94)$. CTC-positive patients were also associated with high recurrence $(\mathrm{OR}=2.84 ; 95 \% \mathrm{CI}=1.81-4.44)$ and poor response of chemoradiotherapy $(\mathrm{RR}=0.64$; 95\% CI=0.43-0.96). For clinicopathological characteristics, CTC-positive patients were significantly associated with TNM staging, depth of infiltration, regional lymph nodes metastasis, distant metastasis, lymphatic invasion, and venous invasion.

Conclusion: The meta-analysis has confirmed the significant clinicopathological and prognostic value of CTC-positive patients for both PFS and OS in patients with EC.

Keywords: esophageal cancer, CTCs, prognosis, meta-analysis

\section{Introduction}

Esophageal carcinoma (EC) is a very most common cancer with the leading cause of death for cancer worldwide. ${ }^{1}$ Although significant progress has been made in diagnostic procedures and multimodality treatment approach of esophageal cancer, more than two-thirds of all patients with EC develop local recurrence or distant metastases. ${ }^{2}$ The recurrence and metastasis supposedly result from clinically occult, minimal residual disease caused by circulating tumor cells (CTCs), or disseminated tumor cells. ${ }^{3}$ CTCs may have the potential to be precursors of metastases and show clinical benefits in treatment and prognosis in patients with esophageal cancer.

CTCs are tumor cells that leave the primary tumor site and enter the bloodstream, where they can spread to other organs. CTCs have been used in many aspects of cancer management, such as monitoring tumor recurrence and treatment efficacy, determining drug-selection strategies, and predicting the survival of cancer patients. ${ }^{4}$ The prognostic value of CTC detection has been confirmed in patients with many cancers, such as lung, ${ }^{5}$ gastric, ${ }^{6}$ and colorectal ${ }^{7}$ cancer. For patients with esophageal cancer, although a previous meta-analysis has shown the clinicopathological and prognostic value of CTCs, ${ }^{8}$ there are some limitations. Most of the retrieved studies in that meta-analysis did 
not show the survival effect, only seven data were available for overall survival (OS), and the prognostic value of CTCs was also unclear in the subanalysis. So there still remains controversy regarding the clinicopathological and prognostic value of CTCs in esophageal cancer.

With the controversies existed in the prognostic role of CTCs for esophageal cancer, here, we conducted the metaanalysis of published literature on this topic to summarize the evidence of the potential clinicopathological and prognostic value of CTCs in esophageal cancer.

\section{Methods}

\section{Search strategy}

Two authors systematically searched the studies. With the keywords "esophageal cancer", "circulating tumor cells", "prognosis", and "peripheral blood", we searched PubMed, EMBASE, Science Citation Index Expanded, and Cochrane library (from inception to July 2018). An additional search through Google Scholar was conducted to identify other potentially relevant publications. Discrepancies were resolved by the third author.

\section{Inclusion and exclusion criteria}

To keep our analysis accurate and reliable, the studies were selected according to the following inclusion and exclusion criteria. The inclusion criteria were 1) studies aiming at the association between the CTCs and either OS or progressionfree survival (PFS) of esophageal cancer; 2) sufficient data to calculate a HR, risk ratio (RR), or OR, and 95\% CI being available; and 3) at least 20 patients were involved in the studies.

The exclusion criteria were 1) studies based on overlapping patients; 2) meta-analysis, review, single test, case report, conference reports, and experiments, reporting of the expert experience; 3) outcome is not clear or the apparent paradox exists.

\section{Data extraction and outcomes}

Data retrieved from the studies included first author's name, year of publication, number of patients, detection rate, country, methods and markers for CTC detection, sample, prognostic value (OS or PFS), HR, etc. Primary outcomes were OS and PFS. Secondary outcomes were overall response rate (ORR), recurrence, and clinicopathological characteristics with CTCs. For studies with multiple markers in CTC detection (each marker within the study can define the positivity of CTCs), each marker was considered an independent data set. If the HR and its 95\% CI for OS or PFS were not reported directly in the original study, available reported data were calculated for the approximated HR using software designed by Tierney et al. ${ }^{9}$ All data were extracted independently by two investigators, and disagreements were resolved by discussion.

\section{Statistical analysis}

We used the STATA 12.0 package (StataCorp, College Station, TX, USA) to analyze the data in our meta-analysis. The estimated HR was used to evaluate the prognostic effect (OS and PFS) as demonstrated by Parmar et al, ${ }^{10}$ and HR $>1$ reflects worse prognosis in the CTC-positive arm. The estimated RR was used to evaluate the response of chemoradiotherapy (ORR), and the estimated OR was used to evaluate the recurrence and summarize the association between CTC detection and clinicopathological characteristics. All statistical values (pooled HR, RR, and OR) were reported with 95\% CIs, and the two-sided $P$-value threshold for statistical significance was set at 0.05 . Heterogeneity was assessed by $I^{2}$ inconsistency test and chi-squared-based Cochran's $Q$ statistic test; $I^{2}>50 \%$ or $P<0.1$ indicated significant heterogeneity. When $I^{2}<50 \%$ and $P>0.1$, the fixed effect model was used, or the random-effects model was used conversely. ${ }^{11}$ Publication bias for survival effects was detected by Begg's test and Egger's test; $P<0.05$ was considered of significant publication bias. ${ }^{12}$ The Newcastle-Ottawa scale was used to assess the quality of the retrieved studies, and score 5-9 was of high quality and score $1-4$ was of low quality. ${ }^{13}$ Furthermore, subgroup analyses were made according to variables such as country, methodology, number of patients, patients' positive rate, and sample. This article follows the Quality Of Reporting Of Metaanalyses (QUORUM) and the Cochrane Collaboration guidelines (http://www.cochrane.de) for reporting meta-analysis (PRISMA statement). ${ }^{14}$

\section{Results \\ Baseline study characteristics}

According to the above-mentioned retrieval method, we identified 431 studies in this systematic literature search. Detailed steps of the search were shown in Figure 1. Finally, 18 cohort studies met the inclusion and exclusion criteria and were included in our meta-analysis. ${ }^{15-32}$ The basic characteristics of these studies were showed in Table 1. A total of 1,719 patients were involved in these studies, the median number of patients was 95, and the median CTC-positive patients rate of the patients was $44.3 \%$ (range from $13.9 \%$ to $69.4 \%$ ). These studies were from five countries (China, Japan, Germany, United Kingdom, and United States) with different detection methods (nine studies used RT-PCT, 


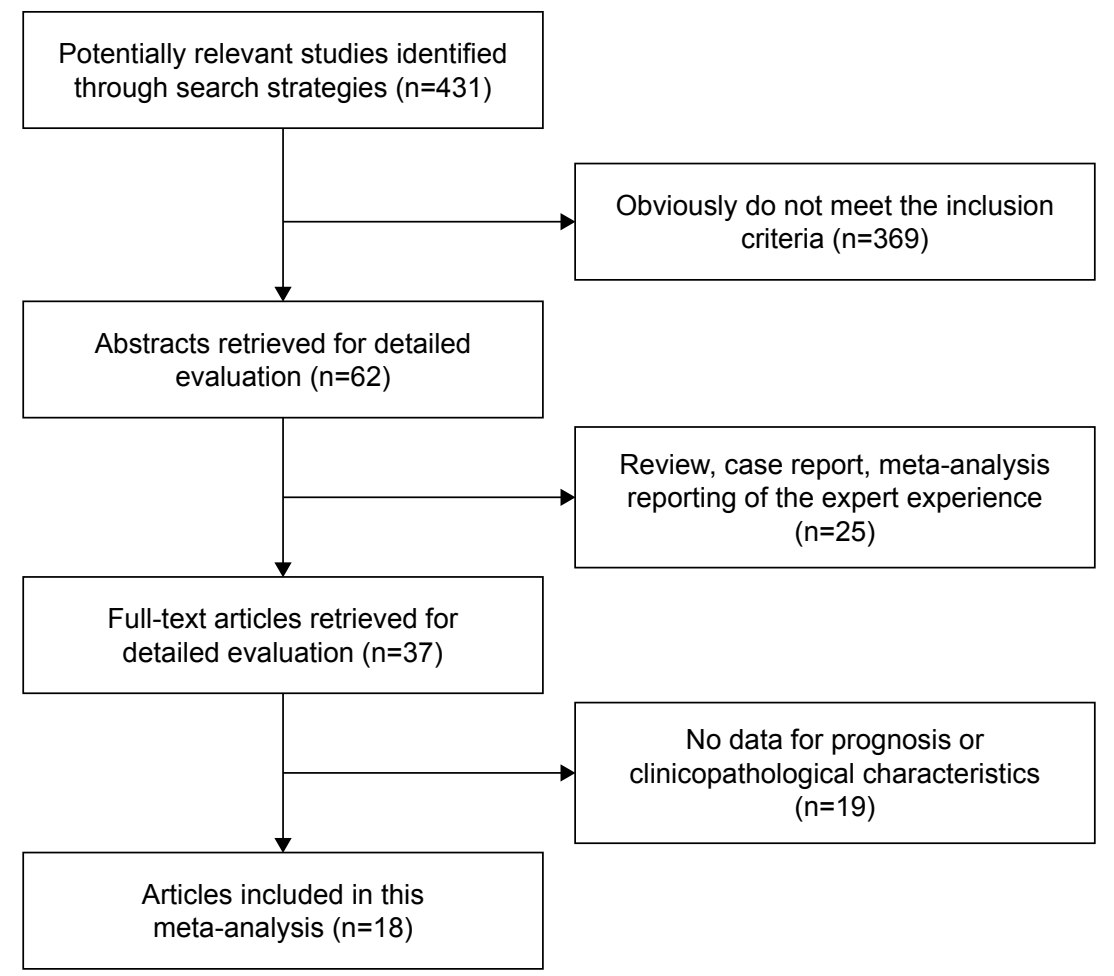

Figure I Selection of the included studies.

while the others used cytological methods). All of the studies provided at least one data for survival effect. For the quality assessment shown in Table 1, all the retrieved studies were of high quality (score 5-7).

\section{OS}

Seventeen studies provided the data for OS with 1,353 esophageal cancer patients. When pooling the HR for OS, no significant heterogeneity between these studies was found

Table I Baseline characteristics and quality assessment by the Newcastle-Ottawa scale of retrieved studies

\begin{tabular}{|c|c|c|c|c|c|c|c|c|}
\hline Study & $\mathbf{N}$ & $\begin{array}{l}\text { Positive } \\
\text { rate (\%) }\end{array}$ & Country & $\begin{array}{l}\text { Detection } \\
\text { methods }\end{array}$ & Markers & Treatment & Outcome & Star \\
\hline Pernot et al, $2017^{30}$ & 106 & 46 & France & CellSearch & EpCAM, CK CD45 & Nonsurgery & PFS, OS & 7 \\
\hline Qiao et al, $2016^{32}$ & 59 & 79.7 & China & Immunofluorescence & CK8/I8/19 CD45 DAPI & Surgery & PFS, OS & 7 \\
\hline Su et al, $2016^{31}$ & 57 & 50.8 & China & Flow cytometry & EpCAM, CK CD45 & Nonsurgery & PFS, OS & 6 \\
\hline Li et al, $2016^{15}$ & 140 & 44.3 & China & $\mathrm{IHC}$ & CKI9 & Surgery & PFS, OS & 5 \\
\hline Reeh et al, $2015^{16}$ & 100 & 18 & Germany & CellSearch & EpCAM, CK, CD45 & Surgery & PFS, OS & 7 \\
\hline Matsushita et al, $2015^{17}$ & 90 & 27.8 & Japan & CellSearch & EpCAM, CK CD45 & Nonsurgery & OS & 6 \\
\hline Kubisch et al, $2015^{18}$ & 62 & 69.4 & USA & $\begin{array}{l}\text { Immunomagnetic } \\
\text { enrichment }\end{array}$ & MUCI, EPCAM & Nonsurgery & PFS, OS & 5 \\
\hline Sclafani et al, $2014^{19}$ & 18 & 44.4 & UK & CellSearch & EpCAM, CK CD45 & Nonsurgery & OS & 6 \\
\hline Ling et al, $2012^{20}$ & 209 & 36.8 & China & PCR & $\mathrm{MSH} 2$ & Surgery & PFS & 5 \\
\hline Yin et al, $2012^{21}$ & 72 & 69.4 & China & RT-PCR & CEA, CKI9 & Nonsurgery & PFS & 5 \\
\hline Song et al, $2012^{22}$ & 85 & 37.6 & China & RT-PCR & STC-I & Surgery & PFS & 5 \\
\hline Hoffmann et al, $2010^{23}$ & 77 & 62 & Germany & PCR & Survivin & Surgery & OS & 6 \\
\hline Tanaka et al, $2010^{24}$ & 244 & 13.9 & Japan & RT-PCR & SCC, CEA & Surgery & PFS, OS & 5 \\
\hline Hoffmann et al, $2009^{25}$ & 59 & 61.0 & Germany & RT-PCR & Survivin & Surgery & OS & 5 \\
\hline Cao et al, $2009^{26}$ & 108 & 47.2 & China & RT-PCR & Survivin & Surgery & PFS, OS & 6 \\
\hline Hiraiwa et al, $2008^{27}$ & 38 & 15.1 & Japan & CellSearch & EpCAM, CK CD45 & Nonsurgery & OS & 5 \\
\hline Setoyama et al, $2007^{28}$ & 125 & 61.6 & Japan & RT-PCR & CEA & Surgery & PFS, OS & 7 \\
\hline Kaganoi et al, $2004^{29}$ & 70 & 32.9 & Japan & RT-PCR & SCCA & Surgery & PFS, OS & 6 \\
\hline
\end{tabular}

Abbreviations: IHC, immunohistochemistry; OS, overall survival; PFS, progression-free survival. 
$\left(I^{2}=18.9 \%, P=0.233\right)$, and a fixed effect model was used. The estimated HR for OS was $2.50(95 \% \mathrm{CI}=2.12-2.94)$, and a poor OS was found in CTC-positive patients compared with the CTC-negative patients. As to the difference of the detection methods, the studies were divided into two subgroups (the PCR group and the non-PCR group). The results were shown in Figure 2. A significant difference in OS between CTC-positive and CTC-negative patients was found in both PCR and non-PCR subgroups. The estimated HR was 2.41 (95\% $\mathrm{CI}=1.83-3.18)$ in the PCR subgroup and 2.54 (95\% CI=2.08-3.11) in the non-PCR subgroup.

\section{PFS}

Fifteen studies with 1,437 patients were available for PFS. As showed in Figure 3, there was a significant heterogeneity in PFS between these studies $\left(I^{2}=50.2 \%, P=0.014\right)$. The random effects model was used, and the pooled HR for PFS was 2.61 (95\% CI=2.08-3.28) (Figure 3). More tumor progression was found in CTC-positive patients than the CTC-negative patients. As to the subanalysis, the heterogeneity in PCR subgroup decreased to no significance $\left(I^{2}=43.5 \%\right.$, $P=0.101)$, and the pooled HR was $2.35(95 \% \mathrm{CI}=1.76-3.15)$ in PCR subgroup and $2.92(95 \% \mathrm{CI}=2.02-4.23)$ in non-PCR subgroup. A poor PFS was found in both subgroups.

\section{Subgroup analyses on survival effects}

Besides detection methods, we made other subgroup analyses according to the difference in the variables (country, number of patients, positive rate of the patients). The results were showed in Table 2. The median patient number per study for OS and PFS was 90 and 108, respectively. And the median positive rate for OS and PFS was $44.4 \%$ and $44.3 \%$, respectively. The results showed a significant prognostic effect for OS and PFS and demonstrated a higher risk of deaths or progression in the CTC-positive patients than the CTCnegative patients for all subgroups. The pooled HRs of OS

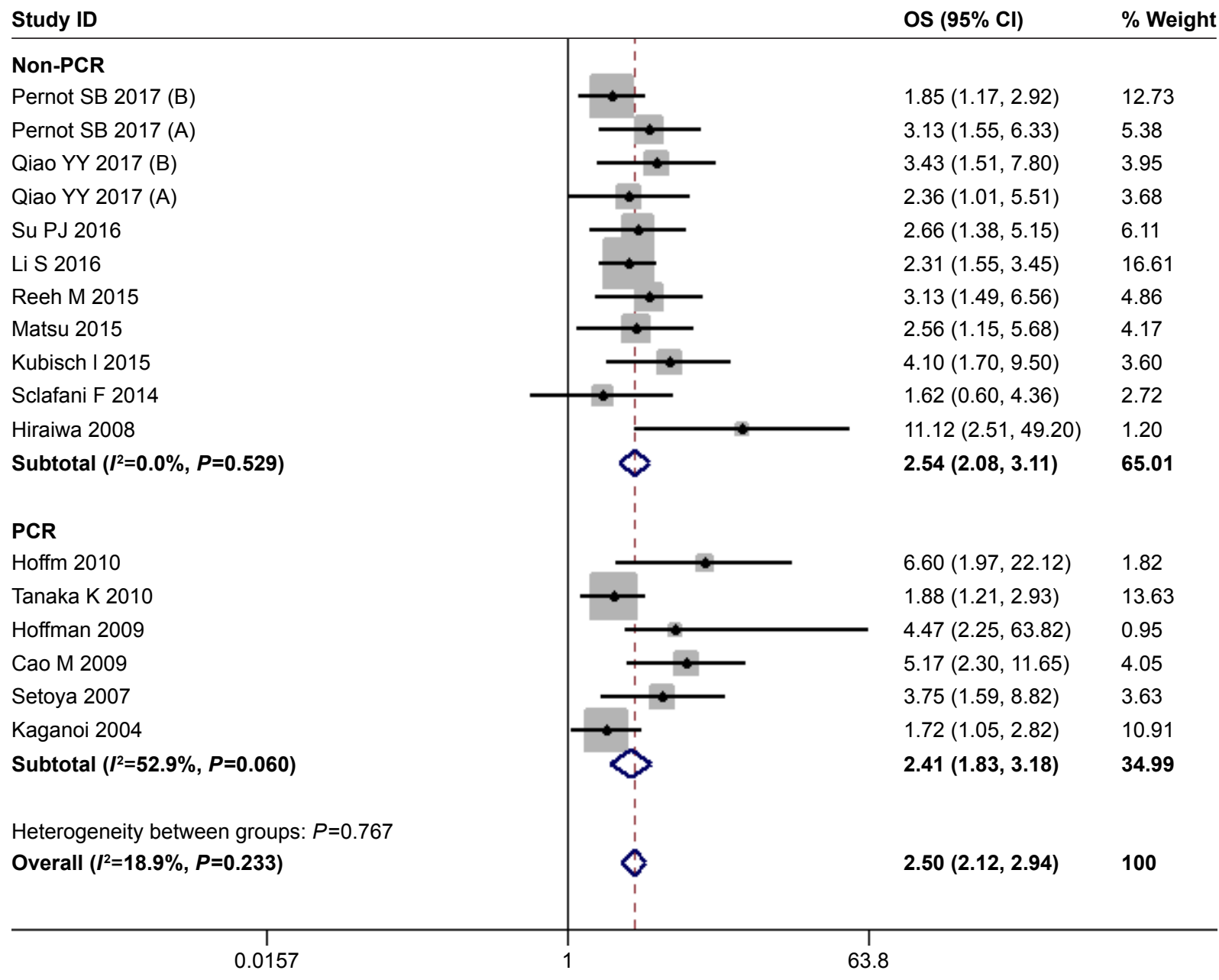

Figure 2 HR for overall survival (OS) of the included studies. 


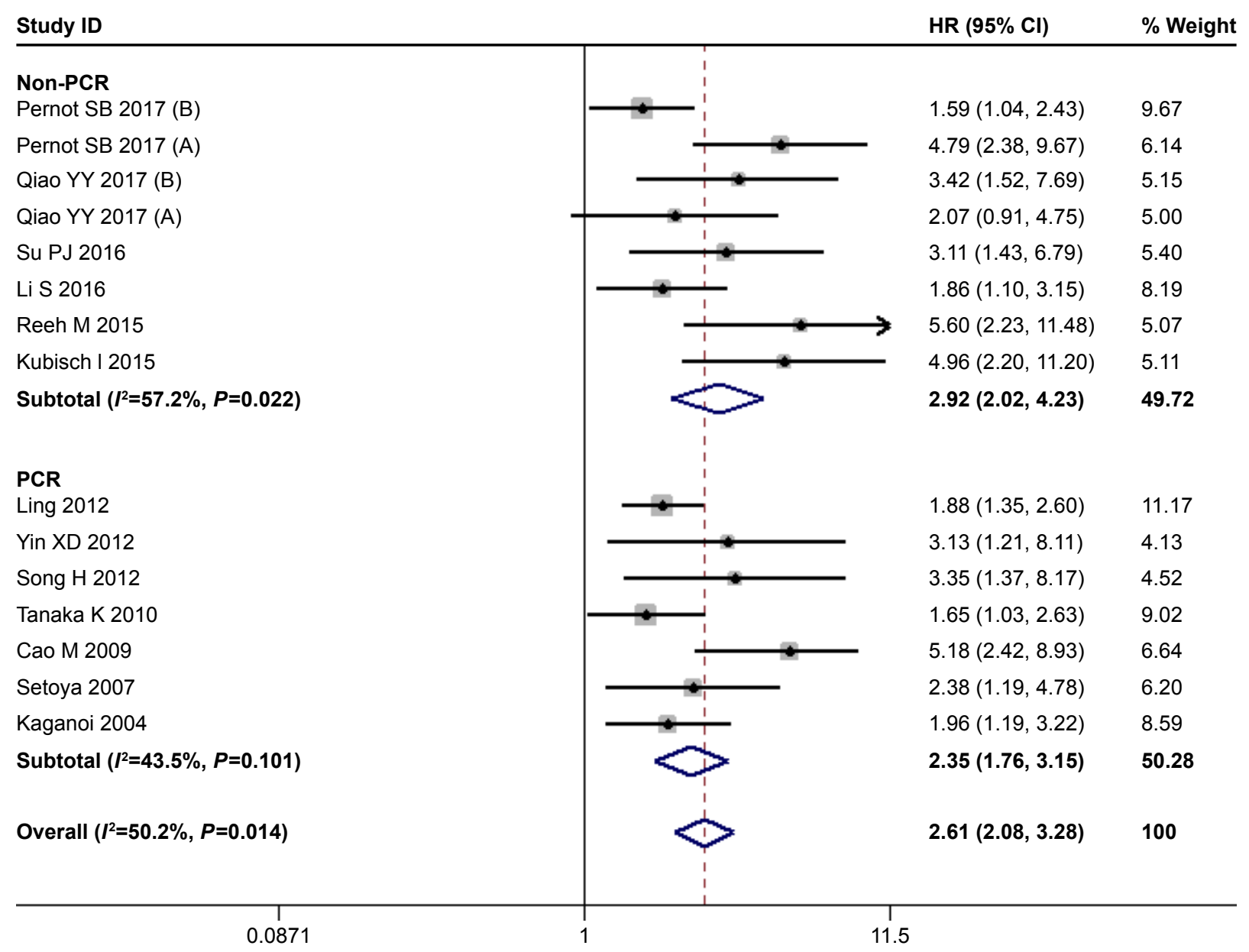

Figure 3 HR for progression-free survival (PFS) of the included studies. Note: Weights are from random-effects analysis.

Table 2 Results of subgroup analyses on PFS and OS

\begin{tabular}{|c|c|c|c|c|c|c|c|c|}
\hline \multirow[t]{2}{*}{ Variables } & \multicolumn{4}{|c|}{ OS } & \multicolumn{4}{|c|}{ PFS } \\
\hline & $\mathbf{N}$ & HR (95\% Cl) & $I^{2}(\%)$ & $P$-value ${ }^{a}$ & $\mathbf{N}$ & HR (95\% Cl) & $I^{2}(\%)$ & $P$-value ${ }^{a}$ \\
\hline \multicolumn{9}{|l|}{ Country } \\
\hline East Asia & 10 & $2.44(2.0 \mathrm{I}-2.98)$ & 27.0 & 0.196 & 11 & $2.3 \mathrm{I}(1.88-2.85)$ & 22.7 & 0.228 \\
\hline Non-East Asia & 7 & $2.61(1.96-3.48)$ & 17.5 & 0.297 & 4 & $3.62(1.77-7.40)$ & 78.2 & 0.003 \\
\hline \multicolumn{9}{|c|}{ Patient no. $\geq$ median $^{\mathrm{b}}$} \\
\hline Yes & 9 & $2.31(1.91-2.80)$ & 31.8 & 0.164 & 8 & $2.37(1.74-3.22)$ & 59.7 & 0.015 \\
\hline No & 8 & $3.06(2.24-4.17)$ & 0 & 0.572 & 7 & $3.00(2.23-4.03)$ & 12.5 & 0.335 \\
\hline \multicolumn{9}{|c|}{ Positive rate $\geq$ median $^{c}$} \\
\hline Yes & 9 & $3.50(2.63-4.64)$ & 0.0 & 0.877 & 8 & $3.55(2.7 \mathrm{I}-4.65)$ & 0 & 0.575 \\
\hline No & 8 & $2.11(1.73-2.58)$ & 10.4 & 0.349 & 7 & $2.01(1.58-2.55)$ & 35.3 & 0.159 \\
\hline \multicolumn{9}{|c|}{ Detection methods } \\
\hline PCR & 6 & $2.4 I(1.83-3.18)$ & 52.9 & 0.060 & 7 & $2.35(1.76-3.15)$ & 43.5 & 0.101 \\
\hline Non-PCR & 11 & $2.54(2.08-3.11)$ & 0 & 0.529 & 8 & $2.92(2.02-4.23)$ & 57.2 & 0.022 \\
\hline Overall & 17 & $2.50(2.12-2.94)$ & 18.9 & 0.233 & 15 & $2.6 \mathrm{I}(2.08-3.28)$ & 50.2 & 0.014 \\
\hline
\end{tabular}

Notes: ${ }^{\mathrm{T} T w o}$-tailed $P$-value of tests for heterogeneity. ${ }^{\mathrm{b}}$ The median patient no. per study for OS and PFS was 90 and 108 , respectively. ${ }^{\mathrm{c}}$ The median positive rate of the patients per study for OS and PFS was $44.4 \%$ and $44.3 \%$, respectively.

Abbreviations: OS, overall survival; PFS, progression-free survival. 


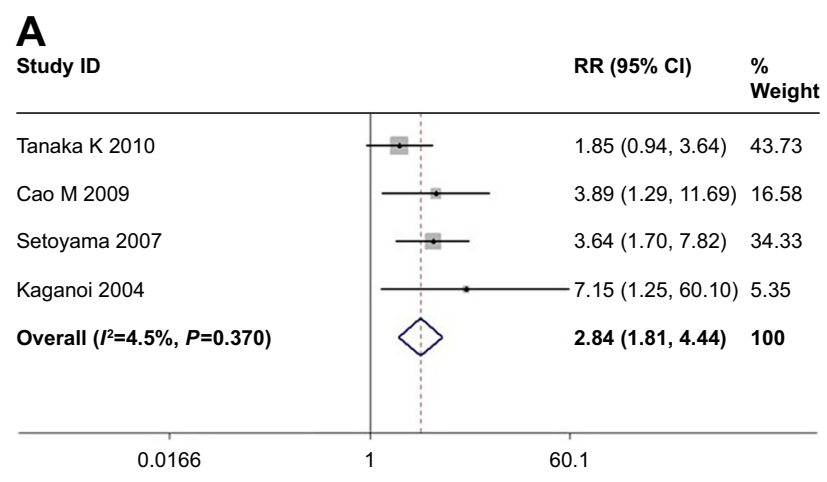

Figure 4 OR for recurrence $(A)$ and risk ratio (RR) (B) for overall response rate. Note: Weights are from random-effects analysis.

and PFS in non-East Asia, non-PCR, high patients' positive rate subgroups were more conspicuous compared with that of their paired subgroups. Besides, for the heterogeneity on OS, it was of no significance in the overall analysis. The heterogeneity in PFS decreased to no significant difference by exclusion of studies in the non-PCR or low patients' positive rate groups, and the obvious heterogeneity in PFS was disappeared in both subgroups when subgroup analyses were made according to the difference in country. This may indicate that country was an important source of inconsistency in PFS.

\section{Recurrence and response of chemoradiotherapy}

Three studies provided the data for the association between tumor recurrence and CTCs. With no significant heterogeneity $\left(I^{2}=4.5 \%, P=0.370\right)$, a fixed effect model was used and the pooled OR was 2.84 (95\% CI=1.81-4.44) (Figure 4A). The pooled OR showed that more tumor recurrence after surgery was found in CTC-positive patients than CTC-negative patients. Tumor recurrence after surgery treatments was more frequently observed in the CTC-positive patients.

For patients who received chemoradiotherapy, the ORR was used to evaluate the response of chemoradiotherapy, and three studies were available. When pooling the estimated $\mathrm{RR}$, a random effects model was used $\left(I^{2}=73.6 \%, P=0.002\right)$; the pooled RR was 0.64 (95\% CI=0.43-0.96) (Figure 4B). A poor response of chemoradiotherapy was found in CTCpositive patients.

\section{Clinicopathological characteristics}

For the clinicopathological characteristics, we extracted the clinical variables from the included studies, when they were mentioned at least in four studies. Then the pooled OR was used to assess the potential correlation between detection of CTCs and clinical variables, and the results were shown

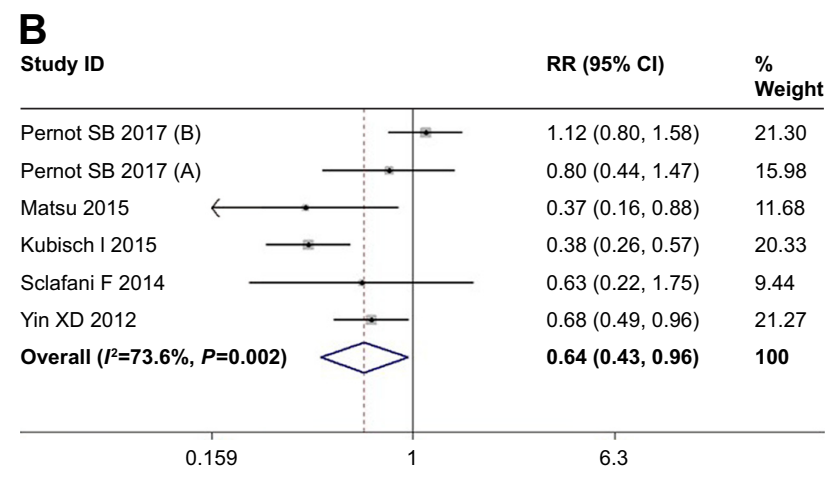

in Table 3. Nine clinicopathological characteristics were extracted and analyzed in our meta-analysis. There were no significant differences of CTCs detection results in gender, degree of tumor differentiation, and tumor location (the pooled OR and 95\% CI was 0.98 [0.70-1.37], 1.20 [0.791.82 ], and 0.82 [0.60-1.11], respectively). However, for other clinicopathological characteristics, 10 studies mentioned the association between pathological stage and CTC detection; the pooled OR indicated a significantly higher incidence of CTCs in the stage III/IV group compared to the stage I/II group, and the OR was 2.51 (1.68-3.76). A same result was also found for the association between CTCs detection and depth of infiltration, regional lymph nodes metastasis, and distant metastasis. Higher incidence of CTCs was found in T3/T4, regional lymph nodes metastasis positive, and distant metastasis positive patients compared with its paired group. Four studies mentioned the association between lymphatic/ venous invasion and CTCs detection. For lymphatic invasion, the pooled OR was $1.73(95 \% \mathrm{CI}=1.17-2.57)$, and for venous invasion, the pooled OR was 2.07 (95\% CI=1.40-3.08). Higher incidence of CTCs was found in either lymphatic invasion or venous invasion positive group.

Table 3 Results of association between CTCs and clinicopathological characteristics

\begin{tabular}{|l|l|l|l|}
\hline Clinical characteristics & OR (95\% CI) & N & P-value \\
\hline Gender (female vs male) & $0.98(0.70-\mathrm{I} .37)$ & $\mathrm{II}$ & 0.889 \\
\hline Grade (G2/3 vs GI) & $\mathrm{I} .20(0.79-\mathrm{I} .82)$ & 9 & 0.130 \\
\hline Location (upper/mid vs lower) & $0.82(0.60-\mathrm{I} . \mathrm{II})$ & 7 & 0.483 \\
\hline Pathological stage (III/IV vs I/II) & $2.5 \mathrm{I}(\mathrm{I} .68-3.76)$ & $\mathrm{II}$ & $0.0 \mathrm{I}$ \\
\hline $\begin{array}{l}\text { Depth of invasion (T3/T4 vs } \\
\text { TI/T2) }\end{array}$ & $2.14(\mathrm{I} .42-3.2 \mathrm{I})$ & $\mathrm{IO}$ & 0.030 \\
\hline Lymph node (NI/N2/N3 vs N0) & $2.33(\mathrm{I} .43-3.79)$ & $\mathrm{II}$ & 0.000 \\
\hline Distant metastasis (yes vs no) & $4.77(\mathrm{I} .77-12.07)$ & 7 & $0.00 \mathrm{I}$ \\
\hline Lymphatic invasion (yes vs no) & $\mathrm{I} .73(\mathrm{I} .17-2.57)$ & 4 & 0.006 \\
\hline Venous invasion (yes vs no) & $2.07(\mathrm{I} .40-3.08)$ & 4 & 0.000 \\
\hline
\end{tabular}

Abbreviation: CTCs, circulating tumor cells. 
Table 4 Results of meta-regression on OS and PFS

\begin{tabular}{|l|l|l|l|l|l|l|l|l|}
\hline \multirow{2}{*}{ Variables } & OS & \multicolumn{4}{l|}{} & \multicolumn{3}{l|}{ PFS } \\
\cline { 2 - 10 } & Coef. & SE & $P$-value & Adj $\boldsymbol{R}^{2}$ & Coef. & SE & $\boldsymbol{P}_{\text {-value }}$ & Adj $\boldsymbol{R}^{\mathbf{2}}$ \\
\hline Country & -0.067 & 0.214 & 0.757 & $-50.21 \%$ & -0.323 & 0.2784 & 0.265 & $-4.97 \%$ \\
\hline Patient no. & 0.281 & 0.201 & 0.059 & $100.00 \%$ & 0.271 & 0.238 & 0.277 & $11.74 \%$ \\
\hline Positive rate & -0.505 & 0.012 & 0.177 & $100.00 \%$ & -0.603 & 0.179 & 0.005 & $100.00 \%$ \\
\hline Methodology & -0.010 & 0.214 & 0.962 & $-59.95 \%$ & -0.180 & 0.245 & 0.477 & $-7.64 \%$ \\
\hline Treatment & 0.001 & 0.210 & 0.996 & $-58.98 \%$ & -0.178 & 0.267 & 0.496 & $-9.27 \%$ \\
\hline
\end{tabular}

Note: Adj $R^{2}$, proportion of between-study variance explained.

Abbreviations: Coef., coefficient; OS, overall survival; PFS, progression-free survival; SE, standard error.

\section{Evaluation of heterogeneity and publication bias}

For the significant heterogeneity in PFS, we conducted a meta-regression to explore the potential sources of heterogeneity, and the meta-regression was made according to the covariates of country, patient number, detection rate, and detection methods. The result was shown in Table 4. In a univariate analysis, the positive rate of the patients showed a significant explanatory variable that influenced the heterogeneity of estimated HR for PFS (coefficient $=-0.603$, $P=0.005$ ), and it explained almost $100 \%$ proportion of between-study variance. Besides, although with no statistical significance $(P=0.277)$, number of patients also explained $11.74 \%$ proportion of between-study variance for the heterogeneity in PFS. For the meta-regression on the heterogeneity of OS, the positive rate of the patients contributed to heterogeneity across studies on OS significantly (coefficient $=-0.505, P=0.177$ ), which explained almost $100 \%$ variances among studies on OS. And country also explained part proportion of between-study variance for the heterogeneity in OS.

Publication bias was detected by Begg's test and Egger's test. Note that, $P<0.05$ meant the existence of publication bias. No publication bias was found in OS (Begg's $P=0.131$, Egger's $P=0.062$ ) and PFS (Begg's $P=0.174$, Egger's $P=0.089$ ).

\section{Discussion}

EC is a malignant tumor originating from the epithelium of the esophagus, and it is one of the most aggressive tumors, with a median survival of less than 2 years and the 5 -year survival rates below $30 \% .{ }^{2}$ CTCs are very important in the diagnosis, treatment, and prognosis of malignant tumors. Lots of studies have been designed to explore the prognostic value of CTCs in many tumors. The poor prognosis of CTCpositive patients has been confirmed in many solid tumors. But for esophageal cancer, the prognostic value of CTCs was still unclear. This meta-analysis provided evidence to evaluate the clinicopathological and prognostic significance of CTCs in patients with esophageal cancer by summarizing all relevant studies.

In this meta-analysis, it has been demonstrated that CTCs in peripheral blood were significantly associated with poor PFS and OS of esophageal cancer patients. CTC-positive patients was a poor prognosis for patients with esophageal cancer. For OS, the risk of death in CTC-positive patients was 1.54 times higher than CTC-negative patients (pooled $\mathrm{HR}=2.50 ; 95 \% \mathrm{CI}=2.12-2.94$ ). For PFS, the risk of tumor progression for CTC-positive patients was 2.62 times higher than CTC-negative patients (pooled HR $=2.61 ; 95 \%$ $\mathrm{CI}=2.08-3.28)$. More death and tumor progression was found in CTC-positive patients compared with CTC-negative patients.

Furthermore, in the subanalysis of the survival effects, our meta-analysis showed a significant prognostic effect for OS and PFS and demonstrated a higher risk of deaths or tumor progression in the CTC-positive patients than the CTC-negative patients in all subgroups. Then, we found that the increased risk of death and tumor progression for CTC-positive patients in non-PCR subgroup was more conspicuous compared with PCR subgroup. Compared with the CTC-negative patients, the increased risk of death and tumor progression for the CTC-positive patients in the two subgroups were 2.68 and 3.53 and 2.41 and 2.35, respectively. Compared with PCR (molecular) methods, non-PCR (cytological) methods could improve the detection specificity, as well as its ability to count CTCs number and recognize viable and functional CTCs. ${ }^{33}$ Taken together, non-PCR methods may provide a more accurate result, which was more conspicuous to detect the increased risk of death and tumor progression in CTC-positive patients. Besides, more conspicuous risk of death and tumor progression in CTC-positive patients was also found in high positive rate subgroup than low positive rate subgroup; this may be because more poor prognostic patients can be identified by using high CTC detection rate methods. 
For the heterogeneity of the pooled survival effects, a significant heterogeneity was found in $\operatorname{PFS}\left(I^{2}=50.2 \%, P=0.014\right)$, we conducted the subgroup analyses and meta-regression to explore the sources of heterogeneity according to the covariates of country, number of patients, patients' positive rate and detection methods. We made subgroup analyses and found that the heterogeneity in PFS dropped to insignificant level when studies were stratified by country. Then in the metaregression, we found that the positive rate had positively contributed to heterogeneity in PFS, and it explained almost $100.0 \%$ proportion of between-study variance. So we finally confirmed the positive rate as the origin of the heterogeneity in PFS. This may be explained by the difference of incidence, mortality, and prognosis of esophageal cancer in different countries. East Asia was the region with the highest incidence and mortality of esophageal cancer in the world. ${ }^{1}$ Besides, we found the patients' number can also contribute to part of heterogeneity in PFS; it explained $11.74 \%$ proportion of between-study variance without statistical significance $(P=0.277)$, so the patients' number may be a potential source of heterogeneity in PFS. For the heterogeneity in OS, there was no significance between the studies; we might think that it was consistent for OS between the involved studies. However, the meta-regression shows that patients' positive rate was of significance $(P=0.049)$ and explained almost $100 \%$ proportion of between-study variance, and the country also explained part proportion of between-study variance in OS. We thought patients' positive rate was potential source of heterogeneity in OS. Finally, we thought that the country and patients' positive rate were potential sources of heterogeneity in our meta-analysis.

Moreover, we assessed correlation between detection of CTCs, tumor recurrence, response of chemoradiotherapy, and clinicopathological characteristics. The CTC-positive patients were easier to occur tumor recurrence after surgery treatments, and gained poor overall response rate of chemoradiotherapy. After escaped from primary tumor and shed into blood, CTCs could be activated due to the loss of biological control by primary tumor and the alteration of internal environment, then CTCs can form the new metastasis or recurrence and cause the resistance of chemoradiotherapy. ${ }^{34}$ The CTC detection can be used to monitor tumor recurrence and chemoradiotherapy efficacy for esophageal cancer, which have been confirmed in other cancers. ${ }^{5-7}$ In addition, our meta-analysis indicated that CTC-positive patients was significantly associated with TNM staging, depth of infiltration, regional lymph nodes metastasis, distant metastasis, lymphatic invasion, and venous invasion. As detection of CTCs is very convenient and comfortable for patients and is repeatable in a noninvasive manner, the presence of CTCs could be used as a monitoring tool for tumor status of esophageal cancer.

There were some limitations in our meta-analysis. First, the meta-analysis used the extracted data, not original data from the individual patients. For the HR and its 95\% CI that we used in our meta-analysis, several data were not provided directly and were estimated from the published data. Second, multiple markers were used to identify CTCs, and the standard for CTC-positive patients in the studies was different. These may contribute to the heterogeneity in our meta-analysis. Third, for the significant heterogeneity in PFS, although the country was the main source of heterogeneity, heterogeneity could not be eliminated because of other factors such as patients' number, patients' positive rate, and detection methods. Therefore, large-scale multicenter studies in homogeneous patients were needed to explore the prognositic value of CTCs.

\section{Conclusion}

Our meta-analysis confirmed the prognostic value of CTCs in esophageal cancer and indicated that CTC-positive patients were associated with poor PFS and OS. CTC-positive patients were also associated with high recurrence and poor response of chemoradiotherapy. CTCs detection can be used to monitor tumor recurrence and chemoradiotherapy efficacy and prognosis for esophageal cancer. For clinicopathological characteristics, CTC-positive patients were significantly associated with TNM staging, depth of infiltration, regional lymph nodes metastasis, distant metastasis, lymphatic invasion, and venous invasion. In future, large-scale multicenter studies by using the same standardized detection platforms are needed to reduce the inconsistencies across studies.

\section{Acknowledgment}

This work was supported by the National Natural Science Foundation of China (Nos. 81302365 and 81302132) and Doctoral Fund of Ministry of Education of China (No. 20130141120059).

\section{Disclosure}

The authors report no conflicts of interest in this work.

\section{References}

1. Torre LA, Bray F, Siegel RL, Ferlay J, Lortet-Tieulent J, Jemal A. Global cancer statistics, 2012. CA Cancer J Clin. 2015;65(2):87.

2. Rice TW, Apperson-Hansen C, Dipaola LM, et al. Worldwide esophageal cancer collaboration: clinical staging data. Dis Esophagus. 2016; 29(7):707-714. 
3. Vashist YK, Effenberger KE, Vettorazzi E, et al. Disseminated tumor cells in bone marrow and the natural course of resected esophageal cancer. Ann Surg. 2012;255(6):1105-1112.

4. Tsujiura M, Ichikawa D, Konishi H, Komatsu S, Shiozaki A, Otsuji E. Liquid biopsy of gastric cancer patients: circulating tumor cells and cellfree nucleic acids. World J Gastroenterol. 2014;20(12):3265-3286.

5. Ma XL, Xiao ZL, Liu L, et al. Meta-analysis of circulating tumor cells as a prognostic marker in lung cancer. Asian Pac J Cancer Prev. 2012; 13(4):1137-1144.

6. Zou K, Yang S, Zheng L, Wang S, Xiong B. Prognostic role of the circulating tumor cells detected by cytological methods in gastric cancer: a meta-analysis. Biomed Res Int. 2016;2016(1):2765464.

7. Huang X, Gao P, Song Y, et al. Relationship between circulating tumor cells and tumor response in colorectal cancer patients treated with chemotherapy: a meta-analysis. BMC Cancer. 2014;14:976.

8. Qiao GL, Qi WX, Jiang WH, Chen Y, Ma LJ. Prognostic significance of circulating tumor cells in esophageal carcinoma: a meta-analysis. Onco Targets Ther. 2016;9:1889-1897.

9. Tierney JF, Stewart LA, Ghersi D, Burdett S, Sydes MR. Practical methods for incorporating summary time-to-event data into meta-analysis. Trials. 2007;8:16.

10. Parmar MK, Torri V, Stewart L. Extracting summary statistics to perform meta-analyses of the published literature for survival endpoints Stat Med. 1998;17(24):2815-2834.

11. Asimit J, Day-Williams A, Zgaga L, Rudan I, Boraska V, Zeggini E. An evaluation of different meta-analysis approaches in the presence of allelic heterogeneity. Eur J Hum Genet. 2012;20(6):709-712.

12. Papageorgiou SN, Papadopoulos MA, Athanasiou AE. Assessing small study effects and publication bias in orthodontic meta-analyses: a metaepidemiological study. Clin Oral Investig. 2014;18(4):1031-1044.

13. Stang A. Critical evaluation of the Newcastle-Ottawa scale for the assessment of the quality of nonrandomized studies in meta-analyses. Eur J Epidemiol. 2010;25(9):603-605.

14. Panic N, Leoncini E, de Belvis G, Ricciardi W, Boccia S. Evaluation of the endorsement of the preferred reporting items for systematic reviews and meta-analysis (PRISMA) statement on the quality of published systematic review and meta-analyses. PLoS One. 2013;8(12):e83138.

15. Li SP, Guan QL, Zhao D, et al. Detection of circulating tumor cells by fluorescent immunohistochemistry in patients with esophageal squamous cell carcinoma: potential clinical applications. Med Sci Monit. 2016;22:1654-1662.

16. Reeh M, Effenberger KE, Koenig AM, et al. Circulating tumor cells as a biomarker for preoperative prognostic staging in patients with esophageal cancer. Ann Surg. 2015;261(6):1124-1130.

17. Matsushita D, Uenosono Y, Arigami T, et al. Clinical significance of circulating tumor cells in peripheral blood of patients with esophageal squamous cell carcinoma. Ann Surg Oncol. 2015;22(11):3674-3680.

18. Kubisch I, de Albuquerque A, Schuppan D, Kaul S, Schaich M, Stölzel U. Prognostic role of a multimarker analysis of circulating tumor cells in advanced gastric and gastroesophageal adenocarcinomas. Oncology. 2015;89(5):294-303.

19. Sclafani F, Smyth E, Cunningham D, Chau I, Turner A, Watkins D. A pilot study assessing the incidence and clinical significance of circulating tumor cells in esophagogastric cancers. Clin Colorectal Cancer. 2014;13(2):94-99.
20. Ling ZQ, Zhao Q, Zhou SL, Mao WM. MSH2 promoter hypermethylation in circulating tumor DNA is a valuable predictor of disease-free survival for patients with esophageal squamous cell carcinoma. Eur $J$ Surg Oncol (EJSO). 2012;38(4):326-332.

21. Yin XD, Yuan X, Xue JJ, Wang R, Zhang ZR, Tong JD. Clinical significance of carcinoembryonic antigen-, cytokeratin 19-, or survivin-positive circulating tumor cells in the peripheral blood of esophageal squamous cell carcinoma patients treated with radiotherapy. Dis Esophagus. 2012;25(8):750-756.

22. Song H, Xu B, Yi J. Clinical significance of stanniocalcin-1 detected in peripheral blood and bone marrow of esophageal squamous cell carcinoma patients. J Exp Clin Cancer Res. 2012;31:35.

23. Hoffmann AC, Vallböhmer D, Grimminger P, et al. Preoperative survivin mRNA detection in peripheral blood is an independent predictor of outcome in esophageal carcinoma. Pharmacogenomics. 2010; 11(3):341-347.

24. Tanaka K, Yano M, Motoori M, et al. CEA-antigen and SCC-antigen mRNA expression in peripheral blood predict hematogenous recurrence after resection in patients with esophageal cancer. Ann Surg Oncol. 2010;17(10):2779-2786.

25. Hoffmann AC, Vallböhmer D, Prenzel K, et al. Methylated DAPK and APC promoter DNA detection in peripheral blood is significantly associated with apparent residual tumor and outcome. $J$ Cancer Res Clin Oncol. 2009;135(9):1231-1237.

26. Cao M, Yie SM, Wu SM, et al. Detection of survivin-expressing circulating cancer cells in the peripheral blood of patients with esophageal squamous cell carcinoma and its clinical significance. Clin Exp Metastasis. 2009;26(7):751-758.

27. Hiraiwa K, Takeuchi H, Hasegawa $\mathrm{H}$, et al. Clinical significance of circulating tumor cells in blood from patients with gastrointestinal cancers. Ann Surg Oncol. 2008;15(11):3092-3100.

28. Setoyama T, Natsugoe S, Okumura H, Matsumoto M, Uchikado Y, Aikou T. Isolated tumour cells in blood and E-cadherin expression in oesophageal squamous cell cancer. Br J Surg. 2007;94(8):984-991.

29. Kaganoi J, Shimada Y, Kano M, Okumura T, Watanabe G, Imamura M. Detection of circulating oesophageal squamous cancer cells in peripheral blood and its impact on prognosis. Br J Surg. 2004;91(8): $1055-1060$.

30. Pernot S, Badoual C, Terme M, et al. Dynamic evaluation of circulating tumour cells in patients with advanced gastric and oesogastric junction adenocarcinoma: Prognostic value and early assessment of therapeutic effects. Eur J Cancer. 2017;79:15-22.

31. Su PJ, Wu MH, Wang HM, et al. Circulating tumour cells as an independent prognostic factor in patients with advanced oesophageal squamous cell carcinoma undergoing chemoradiotherapy. Sci Rep. 2016; 6:31423.

32. Qiao Y, Li J, Shi C, et al. Prognostic value of circulating tumor cells in the peripheral blood of patients with esophageal squamous cell carcinoma. Onco Targets Ther. 2016;10:1363-1373.

33. Beeharry MK, Liu WT, Yan M, Zhu ZG. New blood markers detection technology: a leap in the diagnosis of gastric cancer. World J Gastroenterol. 2016;22(3):1202-1212.

34. Mohme M, Riethdorf S, Pantel K. Circulating and disseminated tumour cells - mechanisms of immune surveillance and escape. Nat Rev Clin Oncol. 2017;14(3):155-167.
OncoTargets and Therapy

\section{Publish your work in this journal}

OncoTargets and Therapy is an international, peer-reviewed, open access journal focusing on the pathological basis of all cancers, potential targets for therapy and treatment protocols employed to improve the management of cancer patients. The journal also focuses on the impact of management programs and new therapeutic agents and protocols on

\section{Dovepress}

patient perspectives such as quality of life, adherence and satisfaction. The manuscript management system is completely online and includes a very quick and fair peer-review system, which is all easy to use. Visit http://www.dovepress.com/testimonials.php to read real quotes from published authors. 